\title{
Stress Analysis of Artificial Knee Joints under Flexion and Rotation
}

\author{
Mitsugu Todo ${ }^{1)^{*}}$, Yuji Takahashi ${ }^{2)}$ and Ryuji Nagamine ${ }^{3)}$ \\ ${ }^{1)}$ Research Institute for Applied Mechanics, Kyushu University \\ 6-1 Kasuga-koen, Kasuga, Fukuoka 816-8580, Japan \\ ${ }^{2)}$ Graduate School, Kyushu University \\ 6-1 Kasuga-koen, Kasuga, Fukuoka 816-8580, Japan \\ ${ }^{3)}$ Yoshizuka Hayashi Hospital \\ 7-6-29 Yoshizuka, Hakata, Fukuoka 812-0041, Japan \\ *Corresponding author: todo@riam.kyushu-u.ac.jp
}

( Manuscript received 12 May 2008; accepted 26 May 2008; published 15 June 2008 )

\begin{abstract}
3-D finite element models of two types of PS type knee prostheses were constructed using their CAD data with use of a nonlinear spring model and an analytical load data for deep squatting. PS-type1 model was formerly used in total knee arthroplasty, and PS-type2 model is the latest version with a modified design of post structure. Stress analysis was then performed by an explicit finite element method under continuous flexion motion from 0 to 135 degrees with internal rotation up to 10 degrees. It was shown that the post/cam contact starts at about 65 degrees of flexion, and the equivalent stress rapidly increases due to the contact. Severe stress concentration is also generated on the Post surface due to the post/cam contact. This kind of stress concentration may cause damage and failure of the post. It was clearly shown that the design modification applied to PS-type 2 effectively reduced the stress concentration of the post.
\end{abstract}

Keywords: total knee arthroplasty, UHMWPE insert, deep flexion, finite element analysis

\section{Introduction}

Total knee arthroplasty (TKA) is applied to patients with severe osteoarthritis as a final treatment to recover the function of the injured knee. After TKA, in general, QOL of the patients is dramatically improved by obtaining knee movement without pain. Although the mobility of knee prosthesis is being improved through design modification, there are still some demands for knee prosthesis such as higher durability of tibial insert. It is therefore needed to understand the detail of movements of operated knee after TKA, and such information should be reflected to the design of knee prosthesis. It is however very difficult to understand the detail of TKA knee motion because of its complex movements characterized as a combination of flexion, rotation and roll-back. Fatigue fracture and severe wear of tibial inserts are sometimes reported, and they are thought to be strongly related to the stress states under such complex knee motions.

PS type knee prosthesis is known to be used in a typical type of TKA where anterior and posterior cruciate ligaments are removed. PS type prosthesis is characterized by the existence of post-cam structure to stabilize the knee movement through post-cam contact.
In this type of prosthesis, failure and wear of the post of the tibial insert are important problems and therefore, there is a demand for understanding the stress state of the tibial insert during knee motion. Under such circumstances, three-dimensional finite element method (FEM) has been utilized to characterize the 3D stress state of knee prosthesis.

In the previous studies of FEM simulations of TKA, most of them were aimed to analyze stress states under walking conditions with shallow flexions ${ }^{1,2-5,8)}$, and a few attempts have been made to analyze stress state under deep flexion $^{9-11)}$. The author's group developed simplified 3D FEM models using CAD data of knee prosthesis clinically used, and investigated effects of deep knee flexion on the stress state of the tibial inserts ${ }^{10,11)}$. In these studies, however, the movement of the tibial inserts of the FEM models was completely restricted and, for example, roll-back behavior in the PS type model was introduced compulsory by moving the femoral component. Recently, a FEM model was developed to reproduce more natural roll-back behavior of PS type knee prosthesis under deep flexional motion $^{12)}$.

In the present study, the FEM model constructed in Ref.12 was extended so that flexional motion is accompanied by internal rotational motion. FEM 
models of two different types of PS type knee prostheses with different post and cam design were developed in order to characterize the effects of the post/cam design on the stress states of the tibial inserts. Effects of internal rotation on the stress states of the tibial inserts were also discussed on the basis of the FEM results.

\section{Development of finite element models}

CAD models of two types of PS type knee prostheses are shown in Fig. 1. These were provided by Stryker's Co. PS-type2, which is clinically used currently, can be recognized as a modified version of PS-type1, and especially, the shape of the post and cam were redesigned to reduce stress concentration. The post of PS-type 2 has more round shape than that of PS-type1, and the contact surface area of the cam to the post was modified to be larger in PS-type 2 than in PS-type1. 3D-FEM models consisting of femoral component, tibial component and tibial insert were constructed from their CAD data.

Finite element meshed models are shown in Fig. 2. Tetorahedral elements were used, and the numbers of the nodes and the elements were 21958 and 89322 for PS-type1 and 28254 and 121604 for PS-type2, respectively. The material constants used in the analysis are shown in Table 1. The tibial insert originally made from a thermoplastic polymer UHMWPE was assumed to be an elastic-plastic material and to follow the von Mises yield criterion. The nonlinear stress-strain relationship experimentally obtained is shown in Fig. $3^{7)}$. The femoral component made from $\mathrm{Co}-\mathrm{Cr}$ alloy and the tibial component made from $\mathrm{Ti}$ alloy are much stiffer than UHMWPE, and therefore, assumed to be rigid body in order to reduce computational time. The friction coefficient between the femoral component and the tibial insert was chosen to be $0.04^{5)}$. It was assumed that the back surface of the tibial insert was perfectly connected to the top surface of the tibial component and therefore, the both surfaces possessed the nodes in common.

In a PS type knee prosthesis attached in a real human knee, reaction and frictional force are generated on the condylar and the Post surfaces during motions. In this TKA knee, these forces are balanced with the tensions of the soft tissues existing around the knee; as a result, for example, roll-back motion occurs. In the present FEA models, a nonlinear spring model was utilized to express these motions in $\mathrm{TKA}^{8)}$. Two spring elements were attached in the front of the tibial component and the two in the back as shown in Fig. 4. The nonlinear force -displacement relation is given by

$$
F=0.18667 d^{2}+1.3313 d
$$

where $F$ and $d$ are force and displacement, respectively. It is noted that this nonlinear relation was experimentally determined from a knee with removed anterior and posterior cruciate ligaments ${ }^{8)}$.
In the most of real knees, internal rotation tends to take place during flexion, and it was reported, for example, that 7 degrees of internal rotation occurs at a deep flexion angle of about 135 degrees $^{13)}$. In the present study, internal rotation of 10 degrees was considered to analyze the effect of such rotation on the stress state of the tibial inserts. The axis of flexion was assumed to be located in the center of the circle which

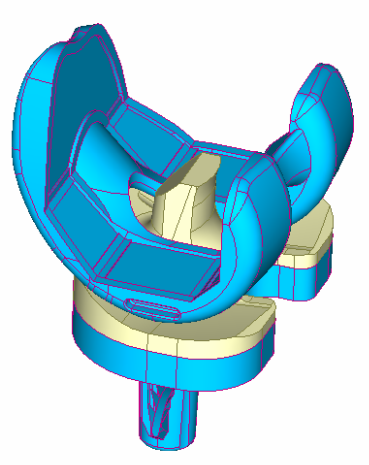

(a) PS-type1

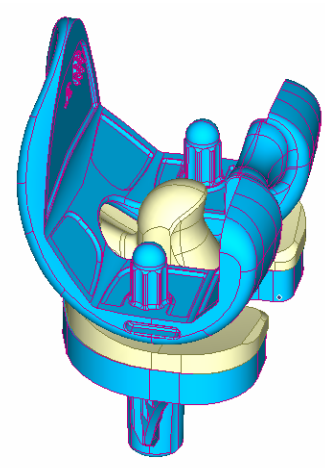

(b) PS-type2
Fig. 1 Two kinds of PS type models

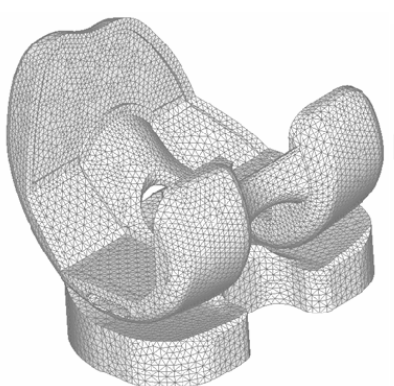

(a) PS-type1

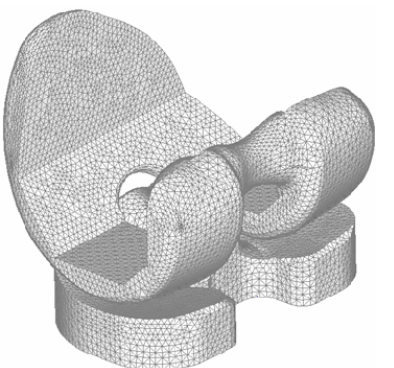

(b) PS-type2
Fig. 2 Finite element meshes

Table 1 Material constants for FEA

\begin{tabular}{ccccc}
\hline \hline Parts & $\begin{array}{c}\text { Density } \\
\left(\mathrm{kg} / \mathrm{m}^{3}\right)\end{array}$ & $\begin{array}{c}E \\
(\mathrm{MPa})\end{array}$ & $v$ & $\begin{array}{c}\sigma_{\mathrm{Y}} \\
(\mathrm{MPa})\end{array}$ \\
\hline \hline $\begin{array}{l}\text { Tibial } \\
\text { insert }\end{array}$ & 940 & 880 & 0.4 & 16 \\
\hline \hline
\end{tabular}

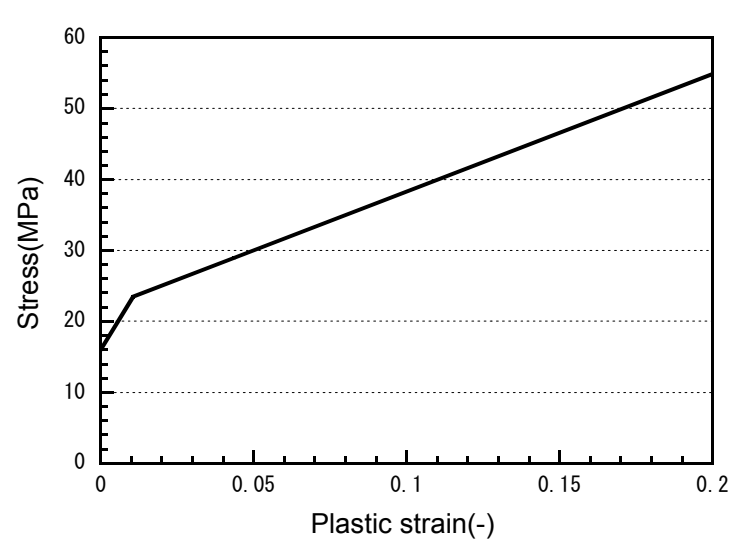

Fig. 3 Stress-plastic strain curve of UHMWPE 


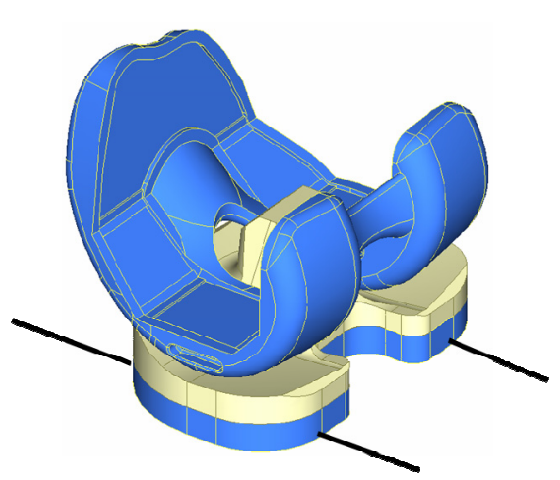

Fig. 4 Simplified model with spring models

coincides with the shape of the condylar surface of the femoral component as shown in Fig. 5. The axis of internal rotation was chosen so that it passes through the center of the tibial component and intersects the axis of flexion. For the femoral component, only the displacement in the Z-direction was free and the tibial component was able to move freely only in the Y-direction (see Fig. 6).

Load data used as the mechanical boundary condition was referred from Ref. 2 in which load data for rapid deep squatting was analytically obtained using 2 dimensional model of human knee considering muscular forces. The load $F_{N}$ and $F_{T}$ were applied to the femoral component in the vertical direction (Z-direction) and to the tibial component in the horizontal direction (Y-direction), respectively, as shown in Fig. 6. The relationship between the load data and the flexion angle were shown with the body force used in the analysis in Fig. 7.

A commercially available pre-processor FE-MAP was used to develop those 3D FEM models including solidification from the surface data, meshing and setting up of the boundary conditions. A commercial explicit finite element code LS-DYNA was then utilized as solver, and a post-processor LS-POST was used to analyze the FEM results.

\section{Results and discussion}

\subsection{Mises equivalent stress distribution}

Von Mises equivalent stress distributions on the surfaces of the tibial inserts at 135 degrees of flexion without internal rotation are shown in Fig. 8. For both models, severe stress concentrations are observed on both the condylar and the post surfaces. Stress is distributed evenly on the right and left condylar surfaces for both the models. It is obvious that the stress concentration on the post surface of PS-type1 is much higher than PS-type2 because the area of the post/cam contact in PS-type1 is smaller than in PS-type2.

Equivalent stress distributions at 135 degrees of flexion with internal rotation are shown in Fig. 9. It is clearly seen that the stress concentration on the left condylar surface is much higher and wider than in the right surface, indicating that internal rotation tends to

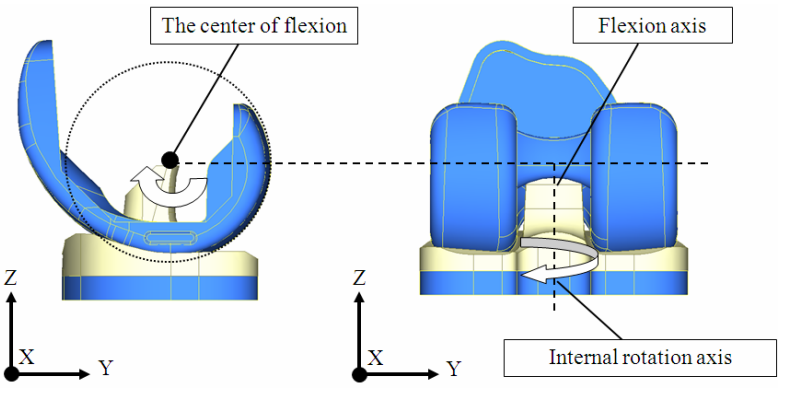

Fig. 5 Axes of flexion and internal rotation

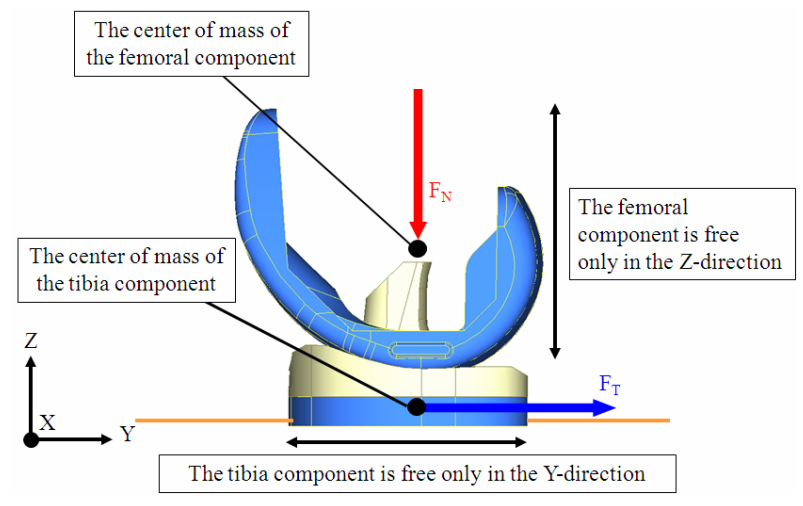

Fig. 6 Boundary conditions

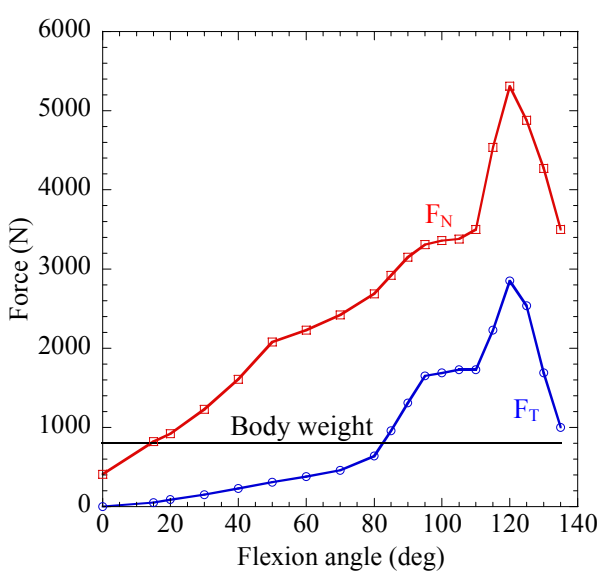

Fig. 7 Analytical force data

increase the stress concentration on the condylar surface and therefore the risk of damage occurrence such as wearing may also increase. The location of the stress concentration on the Post surface moves from the center to the right corner with increase of internal rotation angle. The stress concentration on the post surface of PS-type1 is much higher than PS-type2 because of the sharp edge of the post corner of PS-type1. It is thus concluded that the stress concentration was effectively reduced in PS-type2 at deep flexion angle due to design modification.

3.2. Dependence of maximum equivalent stress on flexion angle

Dependences of the maximum equivalent stress on flexion angle on the posts are shown in Fig. 10. The 'IR' 


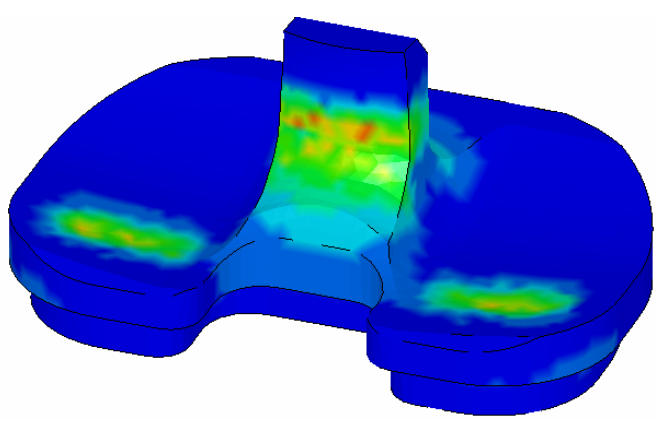

(a) PS-type1

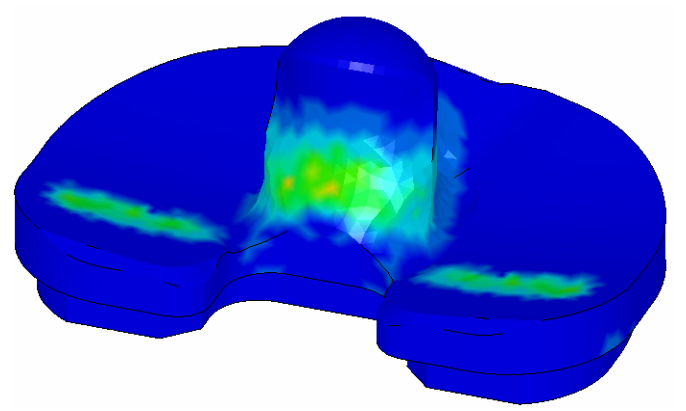

(b) PS-type2

Fig. 8 Equivalent stress concentration at 135 degrees of flexion without internal rotation

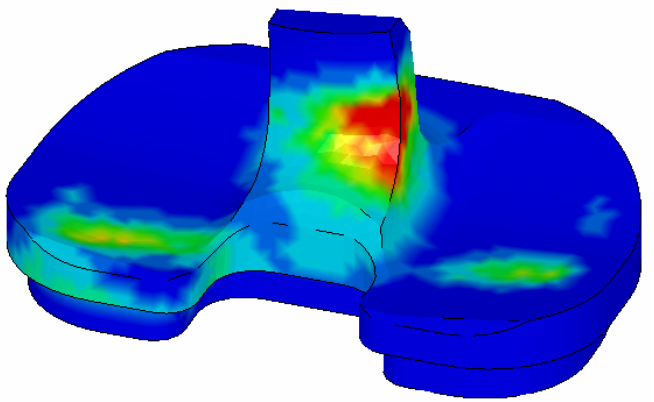

(a) PS-type1

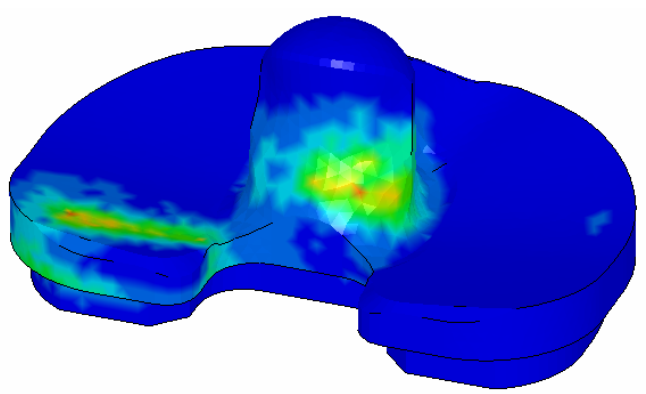

(b) PS-type2

Fig. 9 Equivalent stress concentration at 135 degrees of of flexion with 10 degrees of internal rotation

indicates that the flexional motion was accompanied by internal rotation of 10 degree. Since the maximum stress in the post is usually much higher than that in the condylar part and sometimes causes failure of the post, the post stress is only discussed here. It is obviously seen from Fig. 10 that the difference between PS-type1 and PS-type2 is clearly distinguished. It was found that

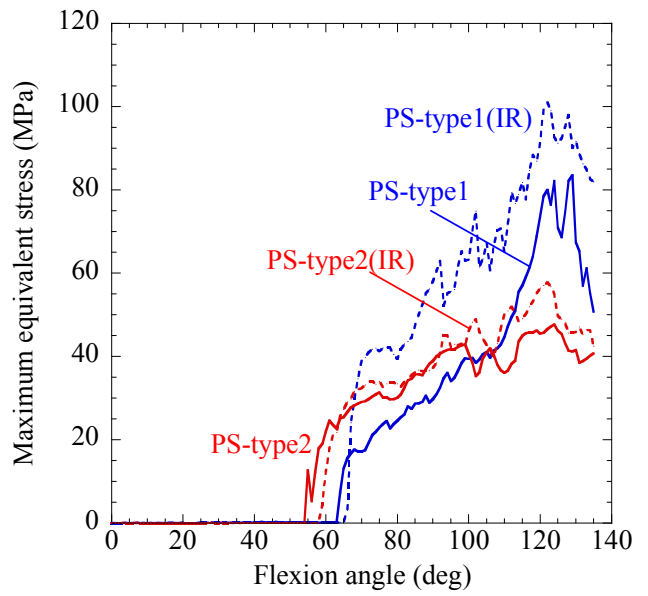

Fig. 10 Dependence of the maximum stresses of the posts on flexion angle

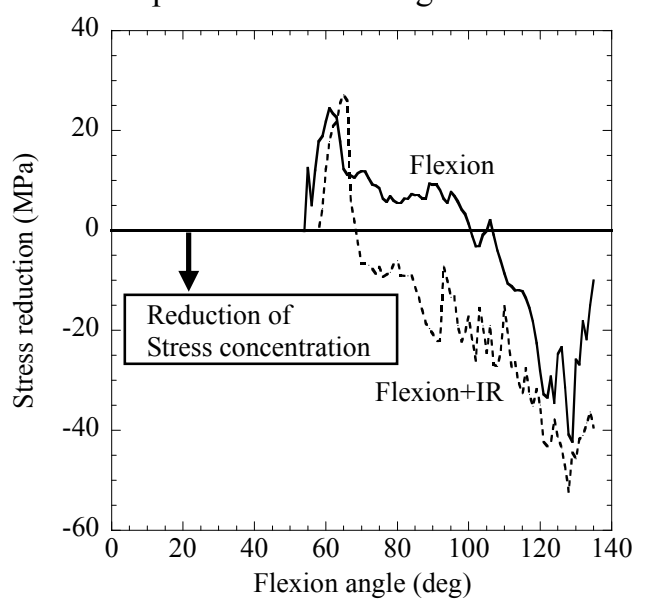

Fig. 11 Stress reduction due to design modification

the post/cam contact starts at about 55 degrees for both the models. The maximum stress in PS-type rapidly increases just after the post/cam contact occurs. The maximum stress reaches its peak at 120 degrees of flexion and the peak values are 100 and $80 \mathrm{MPa}$ with and without internal rotation. It is noted that those stress values are much greater than the yield stress of UHMWPE (16 MPa). It is also important to note that internal rotation increases the maximum stress. Without internal rotation, the maximum stress of PS-type2 is lower than PS-type1 in the flexion range from 100 to 135 degree. For flexion with internal rotation, on the other hand, the maximum stress of PS-type2 is much lower than PS-type1 in the flexion angles larger than 70 degree. It should be noted that the effect of internal rotation is very small in PS-type2. It is thus concluded that the modified design of the post of PS-type2 effectively reduced the maximum stress compared to PS-type1.

Reduction of stress concentration due to the design modification for the post structure is shown in Fig. 11. For only flexion, the post stress tends to increase due to the modified design and is reduced at higher flexion angles than 100 degree. The maximum value of the 
reduced stress is about $40 \mathrm{MPa}$. For flexion with internal rotation, the post stress is effectively reduced at flexion angles greater than 70 degree, and the maximum value of the reduced stress is about $50 \mathrm{MPa}$.

It was found from these analytical results discussed above that the design modification, especially for the post, applied in the model change from PS-type1 to PS-type 2 effectively reduced stress concentration. This kind of reduction of stress concentration generally corresponds to reduction of the reaction force generated by the post/cam contact during deep flexion. Such reduction of the reaction force generally implies the decrease of resistance for deep flexion and therefore, PS-type2 is considered to be more suitable for deep flexion motion than PS-type1.

\section{Conclusions}

3D FEM models of two different types of PS type knee prostheses clinically used worldwide were constructed from their CAD data. Stress states of the two models were analyzed and compared under a condition of deep knee flexion with internal rotation by using the explicit finite element method. The conclusions are summarized as follows:

(1) A simplified 3D-FEA model of PS type knee prosthesis for deep knee flexion analysis including internal rotation was developed by using nonlinear spring model and load data for deep squatting. High stress concentration due to the post-cam contact was reasonably expressed at deep flexion angles and furthermore, roll-back behavior was well simulated by introducing the nonlinear spring model.

(2) Internal rotation tends to increase the stress concentration on both the condylar and the post surfaces, suggesting that the risk of damage formation such as wearing also increases due to existence of internal rotation.

(3) The modification of the Post shape conducted in the designing process of PS-type 2 effectively reduced the stress concentration at deep flexion angles. This kind of stress reduction is thought to correspond to the reduction of reaction force, indicating that the resistance to deep knee flexion is lower in PS-type2 than in PS-type1.

\section{References}

[1] Ahir, S. P., Blunn, G. W., Haider, H. and Walker, P. S., "Evaluation of a Testing Method for the Fatigue Performance of Total Knee Tibial Trays," J. Biomechanics, 32, 1999, 1049-1057.

[2] Dahlkvis, N. J., Mayo, P. and Seedhom, B. B., "Forces during Squatting and Rising from a Deep Squat," Engineering in Medicine, 11, 1982, 69-76.
[3] D’Lima, D. D., Chen, P. C., Kester, M. A. and Colwell Jr, C. W., "Impact of Patellofemoral Design on Patellofemoral Forces and Polyethylene Stresses," J. Bone and Joint Surgery, 85A, 2003, 85-93.

[4] Godest, A. C., Beaugonin, M., Haug, E., Taylor, M. and Gregson, P. J., "Simulation of a Knee Joint Replacement during a Gait Cycle Using Explicit Finite Element Analysis," J. Biomechanics, 35, 2002, 267-275.

[5] Halloran, J. P., Anthony, J. P. and Rullkoetter, P. J., "Explicit Finite Element Modeling of Total Knee Replacement Mechanics," J. Biomechanics, 38, 2005, 323-331.

[6] Kanekasu, K., "Scorpio Superflex Total Knee Arthroplasty-Design, Cilinical Results and Kinematics,” J. Joint Surgery, 23, 2004, 49-57.

[7] Kobayashi, K., Kakinoki, T., Tanabe, Y. and Sakamoto, M., "Mechanical Properties of Ultra High Molecular Weight Polyethylene under Impact Comporession -Property Change with Gamma Irradiation and Dynamic Stress-Strain Analysis of Artificial Hip Joint-," J. The Japanese Soc. for Experimental Mech., 3, 2003, 225-229.

[8] Sathasivam, S. and Walker, P. S., "Computer Model to Predict Subsurface Damage in Tibial Inserts of Total Knees," J. Orthopaedic Res., 16, 1998, 564-571.

[9] Morra, E. A. and Greenwald, A. S., "Polymer Insert Stress in Total Knee Designs during High-Flexion Activities: A Finite Element Study," J. Bone and Joint Surg., Am 87, 2005, 120-124.

[10] Todo, M., Nagamine, R., Kuwano, R., Hagihara, S. and Arakawa, K., "Development of 3D Finite Element Model of Total Knee Arthroplasty and Computational Efficiency," Japanese J. Clinical Biomech., 27, 2006, 231-237.

[11] Todo, M., Nagamine, R., Yamaguchi, S., Hagihara, S. and Arakawa, K., "Effect of Flexion and Rotation on the Stress State of UHMWPE Insert in TKA," Japanese J. Clinical Biomech., 27, 2006, 239-246.

[12] Todo, M., Nagamine, R. and Yamaguchi, S., "Stress Analysis of PS Type Knee Prostheses under Deep Flexion,” J. Biomech. Sci. Eng., 2, 2007, 237-245.

[13] Watanabe, T., Yamazaki, T., Sugamoto, K., Tomita, T., Hashimoto, H., Maeda, D., Tamura, S., Ochi, T. and Yoshikawa, H., "In Vivo Kinematics of Mobile-Bearing Knee Arthroplasty in Deep Knee Bending Motion,” J. Orthopaedic. Res., 22, 2004, 1044-1049. 